\title{
Development of night time temperature rhythms over the first six months of life
}

\author{
M Lodemore, S A Petersen, M P Wailoo
}

\begin{abstract}
Continuous recordings of night time rectal temperature were made at regular intervals over the first six months of life in 49 babies. In the first two weeks of life rectal temperature changed little overnight, but by 6 weeks of age rectal temperature at bedtime was significantly higher than later in the night. By around 12 weeks of age sleeping deep body temperature fell below $36.5^{\circ} \mathrm{C}$, and by 16 weeks of age all babies exhibited a consistent rhythm of rectal temperature. This fell by about $0.8^{\circ} \mathrm{C}$ within two hours of bedtime, and then remained low until an hour or two before waking. As babies got older the mean interval between bedtime and first disturbance of parents got longer. Sleeping rectal temperature fell below $36.5^{\circ} \mathrm{C}$ at about the time babies slept for seven hours. From 6 weeks of age, as individual baby's rectal temperatures fell more with sleep, sleep got longer.
\end{abstract}

By 4 months of age babies exhibit a characteristic pattern of change of deep body temperature at night. It falls from above $37^{\circ} \mathrm{C}$ at bedtime to around $36.4^{\circ} \mathrm{C}$ during the first two hours, remains low for a few hours, and then rises gradually again before waking. ${ }^{1}$ The rapid fall in temperature is associated with sleep so its precise timing is related to bedtime, but the extent of fall is greater during sleeps at night than during the day, even when they are of similar duration. ${ }^{2}$ This suggests that a circadian rhythm has begun to develop, though its expression is still affected by individual sleeping patterns, which may vary considerably from one infant to another at this age.

The rate and extent of fall of temperature is unaffected by ambient temperature and thermal insulating of clothing and wrapping within the range normally chosen by parents ${ }^{3}$; this indicates that the pattern is endogenous and maintained by active thermoregulation if necessary.

This pattern of temperature change does not occur in the newborn, so it must develop during the first few weeks of life. There are some reports that suggest a rhythm appearing around 1-2 months of age, ${ }^{4}$ but there are no reports of continuous recordings on reasonable numbers of babies. In this study, therefore, we have used continuous recording techniques in a combined longitudinal and cross sectional design to observe the development of temperature rhythms in a good sample of babies and have related the temperature patterns to sleep and thermal environment.

\section{Subjects and methods}

Subjects were recruited at birth in the Leicester General and Royal Infirmary maternity hospitals. The purpose of the study was explained to parents and permission sought to make continuous night time recordings of temperature at two to four week intervals over the next six months. If parents agreed basic perinatal data were collected from hospital notes, and in most cases a recording of the baby's body temperature (see below) was made before leaving the hospital.

The subsequent inter-recording intervals for each baby were adjusted so as to try and record from roughly equal numbers of babies in each two week age band from 0 to 24 weeks. Inevitably, some babies were lost to the study. They were replaced with others on which recording started at a later age.

On each recording night the baby was visited at home in the early evening, weighed naked, and temperature probes attached. One soft probe, inserted $5 \mathrm{~cm}$ from the anal margin, measured rectal temperature, and other probes recorded skin temperature on the head and either the abdomen or shin. Only rectal temperature data will be discussed in this paper. A fourth probe measured room temperature at the cot side. All probes were connected to a Grant Squirrell data logger, set to sample at one minute intervals throughout the night. These techniques have been demonstrated to be safe, ${ }^{1}$ and full ethical committee permission was obtained for the study.

Parents were asked to keep a diary of events such as feeds, nappy changes, periods of waking, etc, throughout the period of recording. At each visit a note was made of feeding regimens, and information collected from health diaries kept by parents of illness in the baby and family. At each recording, note was made of items of clothing and wrapping around the baby, so that a thermal insulation, or 'tog value' could be calculated using data supplied by the Shirley Institute, Manchester.

The next day data were down loaded from the data loggers to a computer and scrutinised for evidence of technical problems such as loss of probes. Only unblemished data were analysed further. Mean rectal temperatures, together with standard errors, were collated at various times after bedtime for babies in each two week age band. Statistical comparisons were made using Student's $t$ tests, paired where appropriate. 
Results

SUBJECTS

A total of 49 babies were recruited to the study, and 109 usable temperature recordings were made. Probes were lost during a further seven recordings; these were approximately evenly distributed over the age ranges studied. A total of 41 babies were born after 36 weeks' gestation,

Thermal environment of babies studied. Results are mean (SEM)

\begin{tabular}{|c|c|c|c|c|c|c|c|c|c|c|c|}
\hline & \multicolumn{11}{|l|}{ Age (weeks) } \\
\hline & $\begin{array}{l}0-2 \\
(n=19)\end{array}$ & $\begin{array}{l}2-4 \\
(n=11)\end{array}$ & $\begin{array}{l}4-6 \\
(n=12)\end{array}$ & $\begin{array}{l}6-8 \\
(n=7)\end{array}$ & $\begin{array}{l}8-10 \\
(n=11)\end{array}$ & $\begin{array}{l}10-12 \\
(n=9)\end{array}$ & $\begin{array}{l}12-14 \\
(n=11)\end{array}$ & $\begin{array}{l}14-16 \\
(n=9)\end{array}$ & $\begin{array}{l}16-18 \\
(n=5)\end{array}$ & $\begin{array}{l}18-20 \\
(n=8)\end{array}$ & $\begin{array}{l}20-24 \\
(n=7)\end{array}$ \\
\hline $\begin{array}{l}\text { Tog value } \\
\text { Room } \\
\text { temperature }\left({ }^{\circ} \mathrm{C}\right) \text { : }\end{array}$ & $7 \cdot 2(0 \cdot 78)$ & $12 \cdot 31(1 \cdot 48)$ & $10 \cdot 7(1 \cdot 3)$ & $10 \cdot 08(2 \cdot 3)$ & $11 \cdot 36(1 \cdot 35)$ & $12 \cdot 81(1 \cdot 03)$ & $11 \cdot 7(1 \cdot 5)$ & $11 \cdot 35(2 \cdot 2)$ & $10 \cdot 68(1.9)$ & $10.68(0.96)$ & $11 \cdot 45(1 \cdot 3)$ \\
\hline $\begin{array}{l}\text { Minimum } \\
\text { Maximum }\end{array}$ & $\begin{array}{l}20.45(0.64) \\
23.85(0.52)\end{array}$ & $\begin{array}{l}19.99(1.57) \\
23.95(0.99)\end{array}$ & $\begin{array}{l}18 \cdot 20(0 \cdot 75) \\
22 \cdot 23(0 \cdot 68)\end{array}$ & $\begin{array}{l}18 \cdot 25(0.5) \\
21 \cdot 82(0.54)\end{array}$ & $\begin{array}{l}16 \cdot 82(0 \cdot 62) \\
22 \cdot 12(0 \cdot 62)\end{array}$ & $\begin{array}{l}18.53(0.5) \\
21.59(0.5)\end{array}$ & $\begin{array}{l}14.99(0.92) \\
21.59(0.8)\end{array}$ & $\begin{array}{l}17 \cdot 55(0.8) \\
21.81(0.8)\end{array}$ & $\begin{array}{l}17 \cdot 67(0 \cdot 52) \\
22 \cdot 14(0 \cdot 8)\end{array}$ & $\begin{array}{l}17.98(0.8) \\
22 \cdot 51(0 \cdot 8)\end{array}$ & $\begin{array}{l}15 \cdot 29(0.7) \\
21 \cdot 32(0.6)\end{array}$ \\
\hline
\end{tabular}
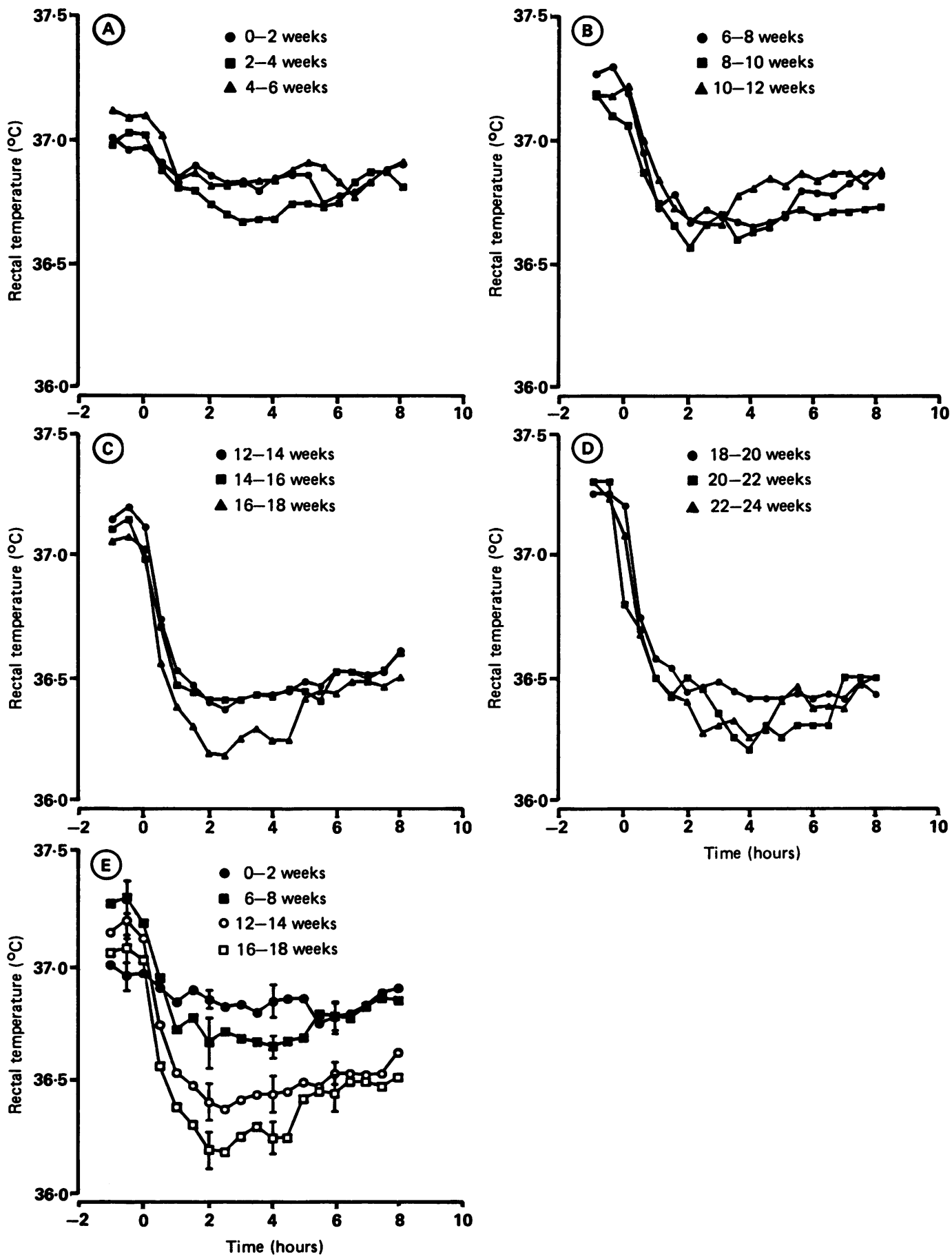

Figure 1 Changes in rectal temperature overnight in babies of different ages. In each case all valid recordings on babies in each two week age band were averaged relative to bediime. Standard errors are mostly not presented on the figures for clarity; they range from $0 \cdot 03-0 \cdot 11^{\circ} \mathrm{C}$, typically $0 \cdot 06^{\circ} \mathrm{C}$. Figures $A$ to $D$ show all data, $E$ shows ages selected to emphasise significant points. 
five between 34 and 36 weeks, and three between 32 and 34 weeks. There were no obvious differences in the data from the small number of babies born prematurely. Twenty three of the babies were recorded on at least four occasions, six recorded three times, and the remainder recorded once or twice to increase numbers in the later age ranges. Most of the recordings over the critical age range of 4-16 weeks formed part of a series of at least four.

\section{THERMAL ENVIRONMENT}

The table shows the minimum and maximum room temperatures and tog values of clothing and wrapping for babies of different ages. The data for babies 0-2 weeks old include some measurements made in hospital (including the special care baby unit occasionally) where ambient temperatures are often high and babies are lightly wrapped. Thereafter all measurements were at home. Babies at home up to 1 month old were kept in slightly warmer rooms, and covered with more insulation, than older babies. From about 6 weeks of age parental behaviour seemed to stabilise, and both room temperatures and tog values did not change significantly as babies got older. These observations were made over a full 12 months, and so contain data from summer and winter, though the seasonal differences were not great.

\section{RECTAL TEMPERATURES}

Averaged data for various ages are presented in fig 1 . In the first two weeks of life rectal temperature varied little overnight, however, by six weeks the temperature was over $37^{\circ} \mathrm{C}$ at bedtime and fell to about $36.8^{\circ} \mathrm{C}$ a couple of hours later. By 14 weeks of age temperature fell with sleep in a pattern like that described previously for babies aged 3-4 months with a minimum rectal temperature below $36.5^{\circ} \mathrm{C}$. This pattern was maintained as babies got older.

Rectal temperature at bedtime was significantly higher in infants aged 6 weeks and older than in those under 6 weeks. By 12 weeks, the body temperature fell significantly further with sleep and produced the 'mature' rhythm.

There were noticeable individual differences in the age at which rectal temperature fell below $36.5^{\circ} \mathrm{C}$ at some time during the night. Some babies achieved this at 6-8 weeks, others not until 14-16 weeks.

\section{TEMPERATURE PATTERNS AND SLEEP}

As we have presented the temperature data relative to the time of placing in a cot, the first hours always corresponded to a period of sleep. In the case of young babies, who slept as much during the day as the night, we recorded night time sleeps only, though we would perhaps expect similar results during the day. At older ages, the first part of the recording usually corresponded to the longest sleep period. We recorded the time between the baby being placed in his or her cot and the first time that the parents were disturbed. Figure 2 shows the mean duration of this sleep for babies of diff-

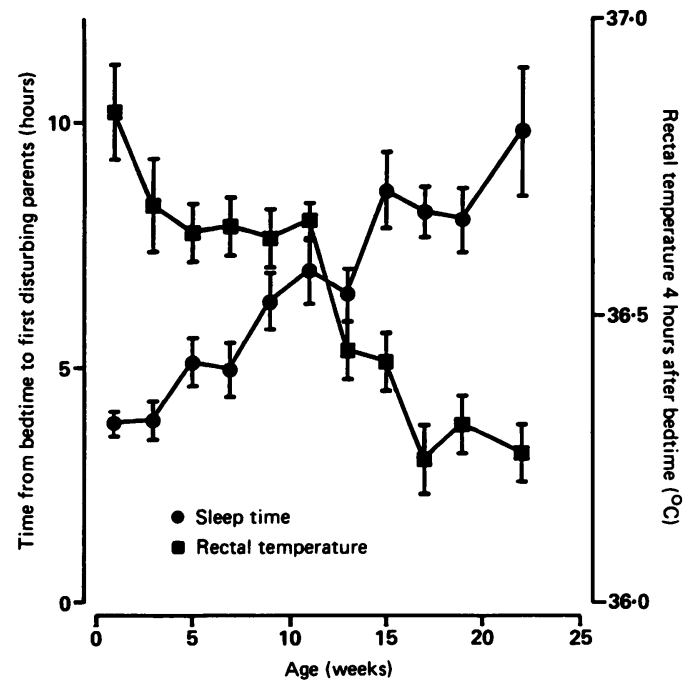

Figure 2 Association between rectal temperature in the middle of the night and age, compared with age related changes in the time from settling in the cot to first disturbance of parents.

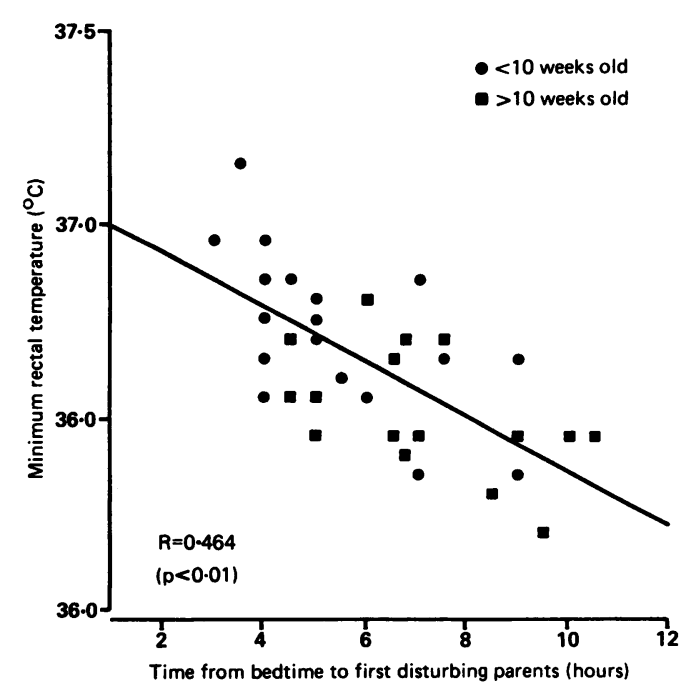

Figure 3 Association between time spent in the cot without disturbing parents and minimum rectal temperature at night for individual babies aged 6-14 weeks. Those whose rectal temperatures fall below $36.5^{\circ} \mathrm{C}$ at an earlier age also sleep for longer earlier.

erent ages compared with the minimum rectal temperature of those babies. As the mean sleep duration increased with age, the minimum nightly rectal temperature fell, though sleep duration increased substantially up to 8 weeks of age with little change in minimum rectal temperature. For individual babies, in the age range 6-16 weeks, however, there was a correlation between the minimum rectal temperature during the first sleep of the night, and the sleep duration (fig $3, r=0.464$, product moment correlation $\mathrm{p}<0.01$ ), suggesting that there was an association between temperature rhythms and sleep rhythms.

\section{Discussion}

We have confirmed our previous observations that a predictable rhythm of night time rectal temperature develops by 16 weeks of age. The development occurs in stages. Babies aged 6 
weeks have higher rectal temperatures than those aged 2 weeks at bedtime. Some weeks later rectal temperature falls more with sleep. There are considerable individual differences in the age at which a minimum rectal sleeping temperature develops, and these are associated with the appearance of longer night time sleeps.

Newborn infants, despite their known limited competence at thermoregulation, ${ }^{5}$ maintain a relatively constant rectal temperature under hospital conditions, which is affected to only a small degree by feeding and sleeping.

Once babies sleep at home parents initially keep them well wrapped under rather warm conditions. By 4-6 weeks of age, however, the parents use a combination of room temperature and wrapping, which varies enormously from baby to baby, to maintain slightly cooler conditions; this is not changed for individual infants as they get older. There are effects of season on parental choice of wrapping and the room temperature but these are small and balanced out by the design of our study.

Despite constant conditions over the first few weeks of life waking rectal temperature, particularly at bedtime, is significantly higher at 6 weeks of age than at 2 weeks of age. This presumably reflects increased activity and increased effects of the feeds. Metabolic rate is known to increase at about this age. ${ }^{6}$

From 4-8 weeks of age sleeping is associated with a temperature fall to about $36.8^{\circ} \mathrm{C}$, as happens during day time sleeps in older infants (and from our few observations at this age as well). By 12 weeks, however, night time sleeps are associated with a larger fall in temperature, and minimum rectal temperature stabilises at around $36.3^{\circ} \mathrm{C}$ by 18 weeks or so. This is a pattern similar to adults. Our data suggest that it continues over the rest of the first year of life, and presumably beyond. All the babies in our study have therefore developed a rhythm of night time temperature fall by 4 months of age. There do, however, appear to be substantial individual differences in the timing of the rhythm development, though the infrequency of our recording does not allow us to pinpoint them with confidence. We are now examining the age range 6-14 weeks in much more detail.

Many other circadian rhythms are developing at this time, ${ }^{4}$ and in particular sleep patterns. There is an association, both on a population and individual basis, between increasing sleep duration and falling minimum rectal temperature. This is not caused by increasing time for cooling as the minimum rectal temperature is attained about the same time in sleeps of widely varying duration, also the temperature pattern is clearly regulated and is unaffected by environmental conditions. We cannot, of course, say whether lower rectal temperature predisposes infants to sleep longer, or rectal temperature falls lower, because the sleep pattern changes. It may just be that independent rhythms of temperature and sleep happen to mature at the same time. It is of interest, however, that the slow wave rapid eye movement sleep pattern changes at about the age that temperature rhythms stabilise.

It would be of considerable interest to correlate the development of temperature rhythm in individual babies with other circadian rhythms in heart rate and hormone secretion. Maybe in some infants there is a critical period around 3 months of age where some rhythms have developed and others have not so that physiological systems become less stable.

1 Wailoo MP, Petersen SA, Whittaker H, Goodenough P. Sleeping body temperatures in 3-4 month old infants. Arch Sleeping body temperatures
Dis Child $1989 ; 64: 596-9$.

2 Anderson ES, Petersen SA, Wailoo MP. Factors influencing the body temperature of $3-4$ month old infants at home the body temperature of $3-4$ month old infants at
during the day. Arch Dis Child 1990;65:1308-10.

3 Wailoo MP, Petersen SA, Whittaker H, Goodenough P. The thermal environment in which $3-4$ month old infants sleep thermal environment in which 3-4 month

4 Minors DS, Waterhouse JM. Development of circadian rhythms in infancy. In: Davies JA, Dobbing J, eds. Scientific foundations of paediatrics. London: Heinemann 1981 980-97.

5 Rutter N, Hull D. Responses of term babies to a warm environment. Arch Dis Child 1979;54:178-83.

6 Azaz Y, Flemming PJ, Levine MR, McCabe R. The relationship between environmental temperature, metabolic rate and sleep state in infants from birth to two months. Early Hum Dev 1989;18:293. 